\title{
Síndrome de Laurence-Moon-Biedl
}

Drs. Vera Wilhelm.* y Víctor Pacheco,*

El Síndrome de Laurence-Moon-Biedl fue descrito por primera vez en 1866 por Laurence y Moon en cuatro pacientes de una misma familia que presentaban talla corta, hipogenitalismo, retardo mental y retinitis pigmentaria. En 1920, Bardet describe un paciente con polidactilia agregado a los hallazgos anteriores y lo incluye en este síndrome. En 1922, Biedl agrega a este síndrome otras malformaciones congénitas, como atresia anal, deformidades del cuello y malformaciones digestivas.

Los aspectos clínicos más característicos de este síndrome son: Obesidad, Retardo mental, Polidactilia, Alteraciones retineales e Hipoplasia genital.

Hasta el año 1973 se han descrito aproximadamente 400 casos.

En los 3 años que lleva funcionando el Policlínico de Endocrinología Infantil del Hospital Clínico Regional de Concepción, hemos tenido la oportunidad de observar 2 pacientes con este síndrome, por lo que hemos creído de interés darlos a conocer.

\section{CASOS CLINICOS}

Caso I: J.E.I. Edad: 4 años 11 meses. Peso: $28 . k g$. Talla: 107,5 $\mathrm{cm}$. Antecedentes familiares: Padres sanos. Dos hermanos: de 3 y 2 años, con desarrollo pondoestatural y psicomotor normales.

Antecedentes personales: Embarazo sin incidentes, no hubo ingestión de medicamentos. Parto de témnino, espontáneo, en su domicilio. Peso de nacimiento: aproximadamente $4.000 \mathrm{~g}$. Sin problemas de asfixia ni ictericia durante el periodo neonatal inmediato. Desarrollo psicomotor: francamente retrasado.

Enfermedades anteriores: Bronquitis a repetición. Crisis convulsivas tónicas acompañadas de opistótonos, pérdida del conocimiento y cianosis peribucal desde 1 año 6 meses de edad; cineo hasta la fecha.

*Departamento de Pediatría, Escuela de Medicina, Lniversidad de Concepción. Policlínico de Endocrinologia Infantil, Hospital Clínico Regional de Concepción.
Enfermedad actual: Consultó en el Policlínico de Endocrinología el 13 de diciembre de 1972, enviada desde un Hospital periférico por sospecha de Hipotiroidismo. Desde los primeros meses de vida ha presentado aumento exagerado de peso. Sus padres notaron evidente retardo psicomotor comparado con sus 2 herinanos. En todo momento el crecimiento estatural fue satisfactorio. Poco activo. Sin intolerancia al frío ni constipación. Sin polidipsia ni poliuria; marcada polifagia. Cefaleas frecuentes. Fatigabilidad fácil.

Examen fisico: Figuras 1, 2 y 3 . Talla normal: $107,5 \mathrm{~cm}$. (entre percentiles 25 y 50). Peso: $28 \mathrm{~kg}$., con sobrepeso de $75 \%$ para su talla. Evidente retardo mental, constatándose un preescolar que sólo dice palabras aisladas, ignora su nombre y no entiende órdenes simples. Abundante panículo adiposo, de distribución unifonne; sin estrías ni acné. Piel húmeda,

Figura 1

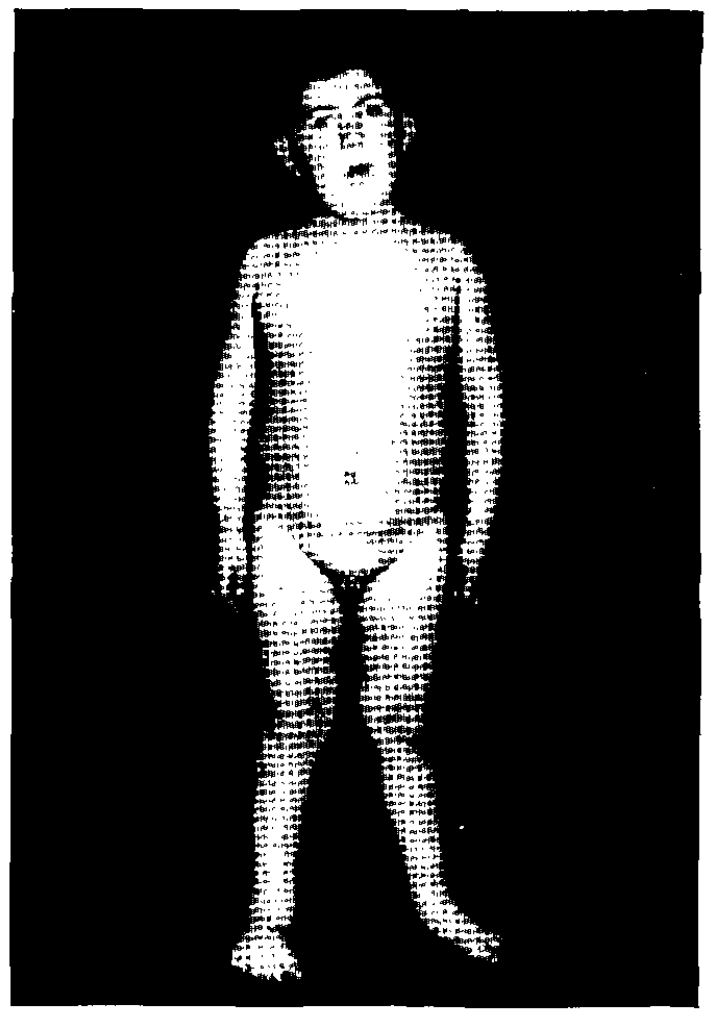




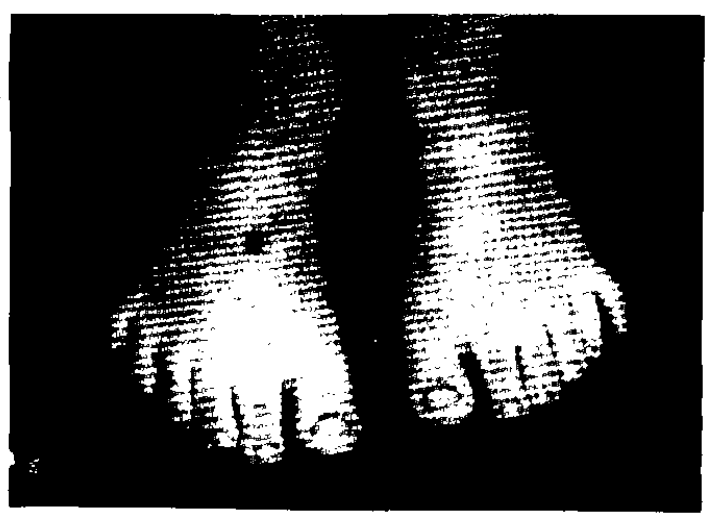

Figura 2

Figura 3

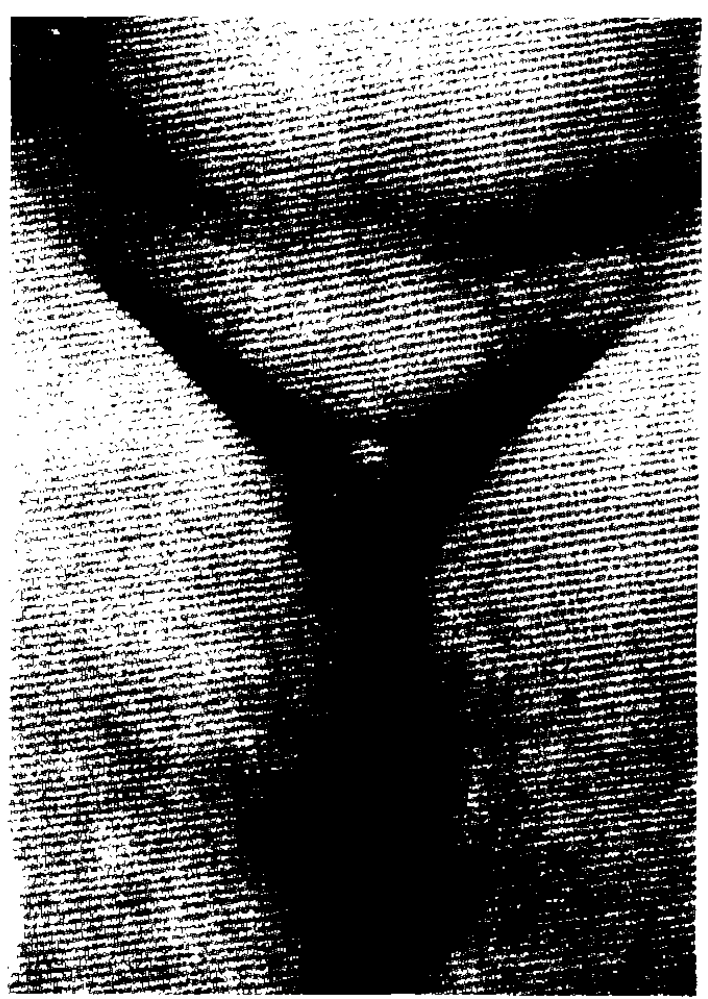

caliente, lina. Fascies vultuosa, con implantación baja de orejas. No hay macroglosia ni mixedema. Paladar ojival, micrognatismo. No se palpa tiroides. Manos anchas, cortas y gruesas, con dedos meñiques incurvados. En los pies hay polidactilia bilateral. Genitales: pene algo pequeño (oculto por tejido adiposo); ambos testículos en el escroto, tamaño de 1-2 inl. (orquidómetro de Prader). Examen neurológico negativo.

Hipótesis diagnóstica: 1. Obesidad; 2 . Hetardo mental; 3. ¿Sindrome de Laurence-Moon-Biedl?; 4. Epilepsia.

Evolución: Este paciente se controló periódicamente en el Policlínico de Endocrinología Infantil hasta la edad de 6 años, durante el lapso de 1 año. La talla se mantuvo entre los percentiles 25 y 50 y el peso, sobre el percentil 97 , con un sobrepeso de $62,5 \%$ a los 6 años de edad, luego de restricción calórica. Én el transcurso de la evolución, sus padres notan además dificultad en la visión.

Exámenes de Laboratorio:

-Ex. oftalmológico: F. de ojo D.I.: retina: aspecto de sal y pimienta con compromiso macular (dispersión del pigmento). Conclusión: Coriorretinitis O.D.I. Astigmatismo.

-E.E.G.: Lesión difusa, predominando immadurez bioeléctrica inespecífica.

-Edad ósea: Entre 4 a 4 1/2 años.

-Radiografía de cráneo: Normal.

-Sedimento urinario: Normal.

El diagnóstico de Síndrome de Laurence-Moon-Biedl en este caso fue confirmado por el hallazgo de coriorretinitis pigmentaria, sumado a la concomitancia de obesidad, retardo mental y polidactilia. Posteriormente, desde principios de 1974, este paciente no concurrió a nuevos controles hasta julio de 1975. En esta oportunidad se constató que había crecido 8,5 cmi, en 1 año, manteniéndose la curva de crecimiento en el percentil 25 y subido sólo $3 \mathrm{~kg}$. de peso en este lapso de tiempo, con lo que el porcentaje de sobrepeso disminuyó a un $59 \%$. Persiste polifagia y discreta polidipsia. El retardo mental se ha hecho más evidente; no concurre al colegio. Se ha acentuado la dificultad en la visión, especialmente ceguera nocturna. Persisten cefaleas frecuentes, pero no ha presentado nuevas crisis convulsivas. En el examen físico no presentaba mayores variaciones con respecto al primer control, llamando la atención que el tamaño de los genitales permanecía igual que hace 1 año.

Caso 2: G.B.F. Figs. 4 y 5 . Edad: 10 años 5 meses. Peso: 53 kg. Talla: $140 \mathrm{~cm}$. Antecedentes familiares: Padres sanos. Cuatro hijos fallecidos en el período de R.N. inmediato. Cuatro hijos vivos; de ellos, una hermana con obesidad y retardo mental severo.

Antecedentes personales: Embarazo sin incidentes y no hubo ingestión de medicamentos. Parto de término, espontá-

Figura 4

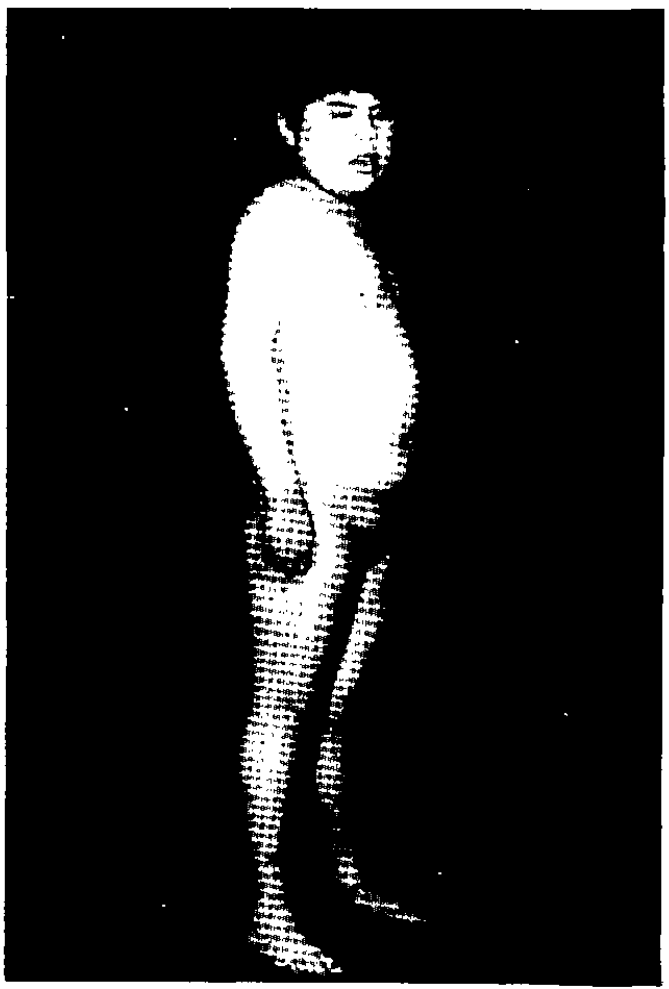




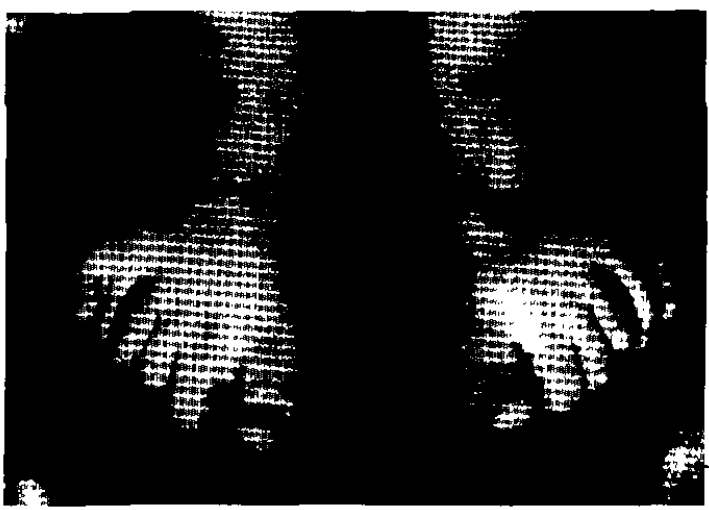

Figura 5

neo, en su domicilio; al parecer, sufrimiento fetal. Peso de nacimiento: aproximadamente $2.000 \mathrm{~g}$. Sin problemas de asfixia ni ictericia durante el periodo neonatal inmediato. Desarrollo psicomotor: muy retrasado: se sentó a los 3 años, se paró y caminó a los 4 años, está en primer afo básico con nulo aprendizaje, dice sólo algunas palabras aisladas.

Enfermedades anteriores: Bronquitis a repetición desde el período de R.N. Sarampión.

Enfermedad actual: Consultó en el Policlínico de Endocrinología Infantil el 9 de mayo de 1974, enviado en interconsulta desde un Hospital periférico por obesidad y retardo mental. Según la madre desde los 6 meses de edad con evidente retardo psicomotor comparado con sus hermanos y desde los 6 años, aumento excesivo de peso. En ningún momento ha dejado de crecer. Discreta polidipsia, sin poliuria, marcada polifagia. Intolerancia al frío discreta y constipación. Desde los 6 años con cefalea y disminución progresiva de la agudeza visual. Tiene una hermana de 14 años que presenta estos mismos síntomas.

Examen flsico: Talla nomal: $140 \mathrm{~cm}$. (en percentil 50 ). Peso: $53 \mathrm{~kg}$. con sobrepeso de 65,2\% para su talla. Evidente retardo mental: dice solo palabras aisladas. Abundante panículo adiposo, de distribución uniforme; sin estrías ni acné. Piel húmeda, caliente, fina. Pulso: $88 / \mathrm{min}$. No se palpa tiroides. Extremidades ligeramente cortas, no hay deformaciones de columna, tórax ancho. Configuración especial de manos, con cicatriz de operación de polidactilia. En los pies hay polidactilia bilateral. Genitales de tipo infantil, ambos testiculos en el escroto de un taman̄o de aproximadamente $2 \mathrm{ml}$. (orquidómetro de Prader). Sin desarrollo puberal.

Hipotesis diagnostica: Síndrome de Laurence-MoonBiedl, fundamentada en la asociación de obesidad, retardo mental, polidactilia y compromiso ocular.

Evolución: Se indicó régimen hipocalórico y se solicitaron exámenes oftalmológico, neurológico, cardiológico, radiografía de cráneo y edad ósea.

Por razones económicas, el paciente no concurrió a los controles ni efectuó los exámenes solicitados.

A requerimiento de la Asistente Social, fue posible controlar a este paciente nuevamente en julio de 1975. Se constató, en esta oportunidad, que había crecido shlo $2 \mathrm{~cm}$. en 1 año 3 meses, pero el peso se había mantenido estacionario, con lo que el porcentaje de sobrepeso disminuyó a un $60,6 \%$.

El retardo mental se ha acentuado, con nulo aprendizaje, se ha acentuado la dificultad en la visión, especialmente ceguera noctuma y aparición de nistagmus. poliuria.

La polifagia habria disminuido. Discreta polidipsia, sin

En el examen físico no se encontraron mayores variaciones con respecto al control anterior, destacando el hecho que el tamaño de sus genitales permanecia igual.

\section{COMENTARIO}

El diagnóstico de Síndrome de Laurence-MoonBiedl se fundamenta en la concomitancia de cinco manifestaciones clínicas: Obesidad, Retardo Mental, Polidactilia, Degeneración retineal e Hipoplasia genital. Cuando estos 5 hechos están presentes, el diagnóstico no ofrece dudas.

Klein y Amman han adoptado la siguiente clasificación:

1. Formas completas, en que están presentes los 5 síntomas cardinales.

2. Formas incompletas, en que pueden faltar 16 2 síntomas cardinales.

3. Formas abortivas, en que sólo hay 162 síntomas cardinales asociados a otras manifestaciones.

4. Formas atípicas, en que la retinitis pigmentaria es remplazada por otras afecciones oculares; por ej.: atrofia optica, oftalmoplejia externa, miopía severa, microftalmia, aniridia y anoftalmia.

5. Formas extensivas, en que además de los 5 síntomas cardinales se suman otras anomalías congéritas o afecciones hereditarias (convulsiones, afecciones espinocerebelares, alteraciones extrapiramidales, etc.).

Estas manifestaciones clásicas pueden estar ausentes en los primeros años de vida y hacerse evidentes con el transcurso de la evolución del paciente, especialmente lo referente a manifestaciones oculares.

Polidactilia: Generalmente se trata de una hexadactilia y se ha observado en un $75-80 \%$ de los casos.

Obesidad: Se describe en un 81-95\% de los casos. Se hace evidente ya en la primera infancia y su severidad aumenta con el transcurso de los años. La distribución del panículo adiposo es uniforme.

Manifestaciones oculares: La retinitis pigmentaria ha sido descrita sólo en un $15 \%$ de los casos. La mayoría de los casos tienen una amplia variedad de manifestaciones oculares atípicas: nistagmus; estrabismo; otras menos frecuentes son microftalmia, anoftalmia, aniridia, atrofia óptica, oftalmoplejia externa, glaucoma juvenil familiar, atenuación de los vasos de la retina, ptosis, coloboma del iris, cataratas, hipertelorismo y miopía. Según algunos autores, las alteracio- 
nes oculares aparecen después de los 5 años de edad; Klein y Amman recalcan que el 73,3\% de sus pacientes estaban ciegos a los 20 años de edad. Entre los síntomas más precoces están presentes la pérdida de la visión central, luego la visión periférica y ceguera nocturna. El diagnóstico de retinitis pigmentaria es difícil en su comienzo por el solo examen de fondo de ojo; la electrorretinografía ha demostrado ser más útil en la detección de la degeneración retineana.

Aspectos endocrinologicos e hipogenitalismo: Según distintos autores como Bell y Klein y Amman, la frecuencia de hipogenitalismo es variable, aproximadamente $74,4 \%$ y $85,7 \%$ respectivamente en hombres, y entre $45 \%$ y $53,1 \%$ respectivamente en mujeres. El desarrollo puberal incompleto y tardío suele ser frecuente.

Se ha descrito, también, asociación con diabetes insípida.

La talla puede ser normal, alta o baja y la edad ósea puede estar acelerada o retrasada.

Otras funciones hipofisiarias parecen no estar afectadas.

Las funciones suprarrenal y tiroidea están normales.

Se ha postulado que el Síndrome de Laurence-Moon-Biedl estaría asociado con hipopituitarismo o con alguna disfunción del diencéfalo o hipotalámica, esto no ha podido ser confirmado.

Desarrollo mental: En un 70-85\% existe retardo mental de distintos grados. Puede hacerse evidente precozmente o más tardíamente con el transcurso de los años. En otros casos se ha descrito repentina pérdida de la función intelectual después de los 7 a 8 años de edad.

En algunos casos se han encontrado anomalías groseras, como atrofia del giro y lóbulos frontales, hidrocefalia, giro cerebral grande y distorsión de la simetría de los hemisferios cerebrales, ausencia de cuerpo calloso y adelgazamiento del mismo. Se hallaron algunas alteraciones microscópicas, como reducción del número de células, anomalías vasculares y gliosis.

Otras anomalias asociadas: Puede existir asociación con sordera, malformaciones renales, cardiopatías congénitas, malformaciones del cráneo y esqueleto (macrocefalia, braquicefalia, hidrocefalia, alteraciones en el tamaño de la silla turca, anomalías de vértebras y costillas, labio leporino y fisura palatina), atresia anal, hipertricosis, aspecto acromegálico y mongoloide.

Alteraciones neurologicas y psiquiátricas:
En raros casos se ha descrito asociación con esquizofrenia, psicosis paranoide, megalomanía, y dislexia. Con mayor frecuencia pueden presentarse hipotonía e hiporreflexia, signos extrapiramidales, degeneración espinocerebelar, espasticidad e hiperreflexia y parálisis de nervios craneanos.

Se han observado frecuentemente anomalías electroencefalográficas tales como: actividad de fondo pobremente organizada y disritmias difusas.

Las convulsiones son poco frecuentes.

Herencia: Klein y Amman piensan que existiría herencia autosómica recesiva, basados en la incidencia familiar en algunas familias suizas estudiadas.

Creemos que en los pacientes que presentamos, el diagnóstico de Síndrome de LaurenceMoon-Biedl queda confirmado por el hallazgo de Obesidad, Retardo mental, Polidactilia y Alteraciones oculares; retinitis pigmentaria en el caso 1 y disminución de la agudeza visual con ceguera nocturna y nistagmus en el segundo caso. Es decir, corresponderían a las formas 1 ó 2 (completas e incompletas, respectivamente) según el criterio de Klein y Amman, ya que por la edad de los niños no podemos evaluar aún el grado de hipogenitalismo.

Es de destacar el hecho que en el segundo de nuestros pacientes existe el antecedente familiar de una hermana de 14 años con el mismo cuadro, que desgraciadamente no hemos podido controlar.

\section{RESUMEN}

Se presentan 2 niños con Síndrome de Laurence-Moon-Biedl.

Se hace una revisión del cuadro clínico y de la bibliografia correspondiente.

\section{SUMMARY}

2 cases of Laurence-Moon-Biedl Syndrome are reported.

The Typical clinical findings of Obesity, Mental Retardation, Polydactylia and Ocular Involvement were present in both patients.

Because of the age we can not yet evaluate the presence of hypogonadism.

\section{REFERENCLAS}

${ }^{1}$ Klein D., Amman F.: Neurol. Sci. 9: 479-513, 1969.

2 Seringe P. et al.: Ann. Endocrinol. 30: 641-659, 1969.

${ }^{3}$ Canivez R.: Ann. Endocrinol. 15: 976-988, 1954.

${ }^{4}$ Ciccarelli E.G., Vessel E.S.: Ann. J.Dis. Child. 101: 519-524, 1961.

${ }^{5}$ Nadjmi B., Flanagan M.J., Christian J.R.: Am. J. Dis. of Child. 117: 352-356, 1969.

${ }^{6}$ Kalbian, V.V.: J. Clin. Endocrinol. 16: 1622-1625, 1956.

${ }^{7}$ Francke C.: J. Clin. Endocrinol. 10: 108-112, 1950.

${ }^{8}$ Bauman, M.L., Hogan, G.R.; Am. J. Dis. of Child. 126: 119 $126,1973$. 